\title{
Parametric Transfer in a Synchronously Pumped Optical Parametric Oscillator
}

\author{
H.S.S.Hung, N.A.Naz, J. Prawiharjo, D.C.Hanna and D.P.Shepherd \\ Optoelectronics Research Centre, University of Southampton, Southampton SO17 1BJ, U.K.,
}

\begin{abstract}
The conditions for high fidelity parametric transfer of the pulse shape of a near-infrared pump pulse to a mid-infrared idler pulse of a synchronously pumped optical parametric oscillator are investigated theoretically and experimentally.
\end{abstract}

\section{Introduction}

Ultrashort mid-infrared (MIR) pulses with controllable phase and amplitude properties have a variety of potential applications in the field of coherent control [1]. Several groups have demonstrated indirect programmable pulse shaping of MIR pulses by pulse shaping in the visible or near-infrared (NIR) followed by difference frequency generation or parametric amplification to reach the MIR [1-5]. In this work, we apply these techniques to a synchronously pumped optical parametric oscillator (SPOPO) by shaping $1.047 \mu \mathrm{m}$ pump pulses, resonating relatively narrow bandwidth $1.5 \mu \mathrm{m}$ signal pulses, and obtaining shaped idler output pulses at $3.4 \mu \mathrm{m}$. We show that the effect of group velocity mismatch (GVM) can adversely affect the fidelity of the transfer and how this can be overcome through cavity length tuning to control the relative timing of the signal and pump pulses.

\section{Modelling of parametric transfer in a synchronously pumped optical parametric oscillator}

In a plane wave approximation, the evolution of the idler spectral envelope, $A_{i}$, in a phase-matched OPO after reaching steady state, is governed by the following equation:

$$
\frac{\partial A_{i}\left(z, \Omega_{i}\right)}{\partial z}=i \gamma \int_{-\infty}^{\infty} A_{p}\left(z, \Omega_{p}\right) A_{s}^{*}\left(z, \Omega_{p}+\Omega_{i}\right) \exp \left[i\left(\delta v_{s i} \Omega_{i}+\delta v_{p s} \Omega_{p}\right) z\right] d \Omega_{p},
$$

where $A_{p}(\mathrm{z}, \Omega)$ and $A_{s}(\mathrm{z}, \Omega)$ are the spectral envelopes of the pump and signal pulses, respectively, $\gamma$ is the nonlinear coupling coefficient, $\Omega_{i, s, j}=\omega-\omega_{i, s, p}$ are detuning coordinates from the carrier frequencies, $\delta v_{s i}$ is the GVM between the signal and the idler pulses, and $\delta v_{p s}$ is the GVM between the pump and the signal pulses. In a sufficiently short nonlinear crystal, the GVM between interacting pulses is negligible, such that the above equation simply reduces to a convolution between the pump and signal pulse spectra, and thus if the signal bandwidth is kept relatively narrow a high fidelity transfer can occur between the pump and the idler [3,4]. However, if the GVM between interacting pulses is not negligible we can see that the $\delta v_{p s}$ term will distort the parametric transfer. For example, if one wished to transfer a chirped pump pulse spectrum to the idler, the GVM would cause the signal and pump pulses to walk away from each other, and thus a preferential transfer to the idler occurs for the part of the pump pulse that has the best temporal overlap with the signal pulse. However, changing the cavity length of an SPOPO offers a possible route for partial compensation of such distortion through altering the relative timing of the pump and signal pulses as shown in the numerical calculations of figure 1. In this simulation the pump pulses were 4ps-wide Gaussians with a phase as typically obtained from self-phase-modulation (SPM) in an optical fibre. A Gaussian filter was introduced inside the cavity such that the signal pulses during steady-state had a spectral FWHM of $40 \mathrm{GHz}$, corresponding to $\sim 9$ ps-wide transform-limited Gaussian pulses. The pump pulses had a peak power of $1.3 \mathrm{~kW}$, leading to a depletion of $\sim 50 \%$. Figure 1(a) shows generated idler spectra as a function of the signal delay with respect to the pump pulses. Figure 1(b) shows the comparison between the idler spectra and spectral phase generated when the signal pulses are delayed by $-0.1 \mathrm{ps}$ with respect to the pump pulse. It can be seen that deliberately delaying the resonated signal pulse, which in this case has the greater group velocity, with respect to the incoming pump pulse leads to a better spectral transfer. As both pulses propagate through the nonlinear medium the signal pulse walks through the pump pulse and so obtains a better temporal overlap with all the spectral components of the pump. The corresponding pulse shape and phase profiles are also optimally transferred at this point. 
(a)

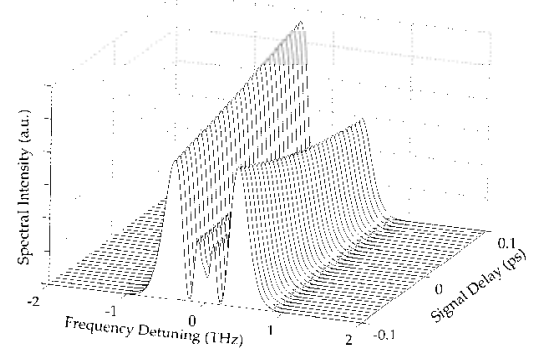

(b)

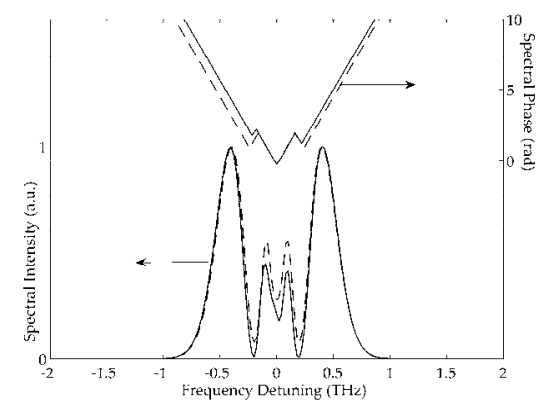

Fig 1(a) Idler spectral intensity against cavity length. Optimum spectral transfer of the symmetric pump spectrum occurs for a signal pulse delay with respect to the pump of -0.1ps. (b) Transfer of the spectral intensity and phase from the pump (dotted lines) to the idler (solid lines) at the optimum cavity length.

\section{Demonstration of parametric spectral transfer in a synchronously pumped optical parametric oscillator}

We experimentally investigated the parametric transfer in a PPLN SPOPO using SPM chirped pump pulses [6]. Figure 2(a) shows how the idler spectrum varied with cavity length tuning, qualitatively confirming the expectation of figure 1(a). The central part of the pump SPM spectrum was removed in this experiment for clarity. Figure 2(b) shows the quality of the spectral transfer at the optimum cavity for the full pump spectrum and with an intracavity etalon limiting the signal bandwidth to $91 \mathrm{GHz}$ with an approximately Gaussian profile.

(a)

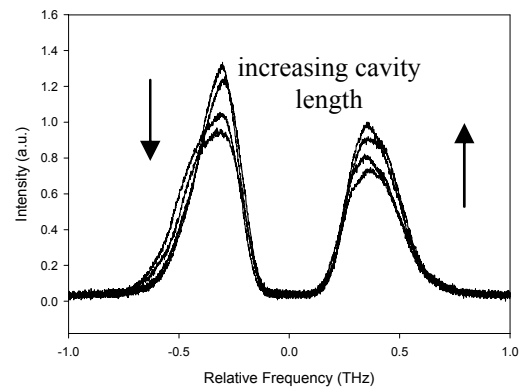

(b)

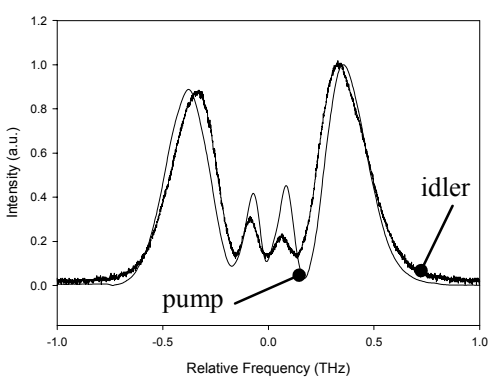

Fig 2(a) Variation of idler spectrum with SPOPO cavity length. (b) Optimum spectral transfer obtained for a signal bandwidth of 91GHz. If the signal bandwidth is not restricted the resolution of the transfer is degraded and the finer features of the spectrum are washed out. Pump depletion for all measurements is $\sim 70 \%$.

\section{Summary}

We have theoretically and experimentally shown that the effect of GVM can distort the parametric transfer from a NIR pump to a MIR idler in a SPOPO. Cavity length tuning is shown to partially compensate for this effect and a relatively good spectral transfer is achieved when the oscillating signal spectrum is restricted with an intracavity etalon, despite high pump depletion. Further numerical and experimental determination of the effect of pump depletion on spectral transfer will be undertaken, along with full phase characterisation via sonogram or FROG techniques.

\section{References}

[1] F. Eickemeyer, R. A. Kaindl, M. Woernaer, T. Elsaesser, and A. M. Weiner, "Controlled shaping of ultrafast electric field transients in the mid-infrared spectral range," Opt. Lett. 25, 1472-1474 (2000).

[2] T. Witte, D. Zeidler, D. Proch, K. L. Kompa, and M. Motzkus, "Programmable amplitude- and phase-modulated femtosecond laser pulses in the mid-infrared," Opt. Lett. 27, 131-133 (2002).

[3] T. Witte, K. L. Kompa, and M. Motzkus, "Femtosecond pulse shaping in the mid infrared by difference-frequency mixing," Appl. Phys. B 76, 467-471 (2003).

[4] H. -S. Tan, E. Schreiber, and W. S. Warren, "High-resolution indirect pulse shaping by parametric transfer," Opt. Lett. 27, 439-441 (2002).

[5] H. -S. Tan, and W. S. Warren, "Mid infrared pulse shaping by optical parametric amplification and its application to optical free induction decay measurements," Opt. Express 11, 1021-1028 (2003), http://www.opticsexpress.org/abstract.cfm?URI=OPEX-11-9-1021.

[6] N.A. Naz, H. S. S. Hung, M. V. O'Connor, D. C. Hanna, and D. P. Shepherd, "Adaptively shaped mid-infrared pulses from a synchronously pumped optical parametric oscillator," Opt. Express 13, 8400-8405 (2005), http://www.opticsexpress.org/abstract.cfm?URI=OPEX-13-218400 . 